\title{
CLIMA ORGANIZACIONAL RELACIONADO CON LA MOTIVACIÓN DEL PROFESIONAL DE ENFERMERÍA DEL HOSPITAL REGIONAL DE ICA 2017 - 2018
}

\author{
Organizational climate related to the motivation of the nursing professional at the Regional Hospital \\ of Ica $2017-2018$
}

Dra. Zulema Gutiérrez Lazo de la Vega $a^{1, a, b, c, d}$

1. Hospital Regional de Ica.
a. Lic en Enfermería, ${ }^{b}$ Enfermera Especialista en Enfermería Materno Infantil con Mención en Ginecología, ${ }^{c}$ Mg. en
Educación con mención en Administración y Planificación de la Educación Superior, ${ }^{d}$ Doctorado en Salud Publica.

\section{RESUMEN}

El presente estudio tiene como Objetivo: la relación del clima organizacional con la motivación del profesional de enfermería del Hospital Regional de Ica 2017 - 2018.Material y métodos: El diseño del estudio Observacional, descriptivo - correlacional, aplicada y cuantitativa. Resultados: muestran que el clima organizacional y su relación con la motivación de los profesionales, de los que tienen una motivación desfavorable (29 profesionales de enfermería), el 10,3\% califican al clima organizacional como medianamente favorable y la mayoría califica al clima organizacional como desfavorable representando el $89,7 \%$. Similar pasa en los profesionales que tienen una motivación favorable ( 7 profesionales de enfermería), el $42,9 \%$ califican al clima organizacional como favorable y medianamente favorable. La correlación entre el clima organizacional con la motivación del profesional de enfermería tiene una correlación directa y alta con un valor de 0,001 por lo que se comprobaría la hipótesis específica. Conclusiones: El estudio realizado en nuestra institución según se refleja en las dimensiones en cuya respuesta señala que el clima organizacional en el Hospital Regional de Ica se encuentra en un nivel bajo es decir que no es favorable, por lo que los administradores tienen un buen margen para mejorar esta variable. En relación a las dimensiones de esta variable todas guardan relación con la motivación del profesional significativamente.

Palabras claves: Clima Organizacional, motivación, profesional de enfermería.

\section{SUMMARY}

The present investigation was carried out with the objective of determining the relation of the organizational climate with the motivation of the nursing professional of the Regional Hospital of Ica 2017 - 2018. Material and Methods: It is a quantitative - correlational descriptive study, for the collection of the data the instruments were used a survey of organizational climat e and a motivational survey, the surveys were applied to 58 nursing professionals of the Regional Hospital of Ica. Results: The results show that the organizational climate and its relation with the motivation of the professionals, of those who have an unfavorable motivation (29), 10.3\% qualify the organizational climate as moderately favorable and the majority qualifies the organizational climate as unfavorable representing the $89.7 \%$. Similar happens in professionals who have a favorable motivation (7) $42.9 \%$ qualify the organizational climate as favorable. The correlation between the organizational climate and the motivation of the nursing professional has a direct and high correlation with a value of 0.001 , for which the specific hypothesis would be verified. Conclusions: The organizational climate in the Regional Hospital of lca is at a low level that is to say that it is not favorable, so that administrators have a good margin to improve this variable. In relation to the dimensions of this variable all relate to the motivation of the professional significantly.

Keywords: Organizational Climate, Motivation, Nursing Professional. 


\section{INTRODUCCIÓN.}

El proceso de modernización del estado peruano abarca a todas las instituciones a nivel nacional, incluidas también las instituciones de salud, este proceso abarca dos finalidades, la optimización de la gestión pública basada en un estado democrático, descentralizado y la de generar recursos económicos, científicos y sociales.

La cultura organizacional, el clima organizacional y la satisfacción del personal son componentes importantes en el comportamiento cotidiano de los profesionales de salud y por consecuencia parte importante del proceso de modernización del aparato estatal.

Por otro lado, los hospitales son organizaciones públicas, en estos trabajan personas dentro de un espacio delimitado atendiendo personas que en su mayoría tienen problemas de salud, bajo esta lógica evidenciamos que estos constituyen un espacio estructurado de prácticas médicas y no médicas con una adecuada coordinación y segmentación del trabajo, este trabajo se desarrolla en determinadas condiciones laborales, estás según muchos autores son factores determinantes de éxito en toda organización (1). En este sentido haciendo una recopilación bibliográfica al respecto, estudios ejecutados como: en Argentina por Vera M. en el año 2013 titulado: Clima organizacional de enfermería en los hospitales regionales del Instituto de Previsión Social, se realizó en base a parámetros cuantitativos, descriptivos y analíticos, los resultados reflejan satisfacción en los siguientes factores: Especialidad donde cumplen funciones cada una de las enfermeras y la imagen gerencial de la enfermera jefe. Revelaron insatisfacción en infraestructura, falta de cursos de capacitación como oportunidad para el desarrollo profesional y personal, inequidad salarial y falta de alcance de beneficios económicos adicionales al salario, el clima organizacional en los hospitales regionales se percibe, en general como positivo porque existen posibilidades de modificar factores que favorecen una mejora en las condiciones laborales. (2), son "factores determinantes de éxito en toda organización". El estudio en Trujillo en el 2015 por Chávez D, Ríos D. Investigación sobre Clima Organizacional y satisfacción laboral de las enfermeras en el servicio de Cirugía del Hospital Víctor Lazarte Echegaray concluyen, que el Clima organizacional tiene relación altamente significativa con la satisfacción laboral según la prueba de Pearson $P=0.76$. (3). En investigaciones locales, como las realizadas por Del Rio J, Munares A, Montalvo H. 2013, en su estudio sobre el clima organizacional en trabajadores de un hospital general de Ica, en el que incluyo 7 grupos ocupacionales, con un total de 178 trabajadores, seleccionados mediante muestreo aleatorio proporcional a grupos ocupacionales; cuyo resultado fue que el clima organizacional percibido por los trabajadores del Hospital tuvo un puntaje promedio de 164, es decir un clima por mejorar. El 12,9\% de trabajadores percibió un clima saludable. De las 11 dimensiones estudiadas, el clima que se percibió fue por mejorar .Las conclusiones de este estudio claramente indican que es necesario mejorar el clima organizacional de la institución, aplicando un plan de intervención con proyectos de mejora del entorno organizacional.(4), de la misma manera se puede observar en la investigación ejecutada por Melgar D.2016.,Clima organizacional en los licenciados de enfermería en el Hospital San José de Chincha, la muestra estuvo conformada por 50 enfermeros asistenciales, el $94 \%$ son de sexo femenino, el $82 \%$ son nombrados( personal estable);con respecto a la variable clima organizacional, según la dimensión comunicación en el $70 \%$ es medianamente favorable, en la dimensión confort el $66 \%$ es medianamente favorable ; llegando a la conclusión que el clima organizacional en la institución investigada es medianamente favorable (5), existiendo concordancia con la investigación realizada 
por Del Rio J, Munares A, Montalvo H (4). Un adecuado conocimiento del clima organizacional por los que dirigen las instituciones y los trabajadores, alcance real importancia al formar parte de las organizaciones que pretenden ostentar altos rendimiento y niveles de eficacia.

El comportamiento de un trabajador, no es el resultado directo de los factores organizativos existentes, sino que dependen en buena medida de la valoración que el trabajador hace de estos factores con las actividades, interacciones y otras experiencias del colectivo con la institución, y que son reflejadas en los estudios del clima organizacional (6). Por lo tanto, al abordar el componente de Recurso Humano como personal médico, enfermería y administrativos es crucial la interrelacionar clima organizacional y motivación laboral; siendo ideal encontrar una alta relación entre el clima organizacional y satisfacción laboral, o al menos que reporten niveles medios en ambas variables (7).

En relación a lo analizado es de importancia el abordaje del estudio, porque los datos estadísticos reportados, permitirá orientar a elaborar estrategias para fortalecer si fuera necesario el clima organizacional en el área de enfermería, que haga construir una personalidad propia coherente, en los nuevos desafíos, proporcionada además de motivación personal por el cuidado cálido y humano, que ofrezca satisfacción al usuario. El estudio tuvo como objetivo determinar la relación del clima organizacional, con la motivación del profesional de enfermería del Hospital Regional de Ica 2017 - 2018.

\section{MATERIAL Y METODOS.}

El presente trabajo de investigación es de tipo de corte transversal, porque se recogió la información en un determinado momento, haciendo un corte en el tiempo, con el propósito de describir las variables, para analizar su incidencia y diseño cuantitativo, descriptivo de corte transversal.
La población. Lo constituyeron 290 enfermeros/as de los diferentes servicios del Hospital Regional de Ica

Que se desempeñan en las funciones asistenciales. La muestra se hizo uso del muestreo probabilístico aleatorio, en donde se considerara intencionalmente al profesional de enfermería de la población que cumpla con las características para el estudio, conformada por 58 profesionales de enfermería utilizo como técnica de recolección de datos la entrevista y la encuesta, como instrumento se utilizó un cuestionario dirigido y estructurado en base a instrumentos validos a través del juicio de tres expertos en la temática investigada ,que permitió recoger información acerca del clima organizacional y la motivación de los profesionales de enfermería y la confiabilidad, Alfa de Cronbach fue del 95\%de confianza ,con un error muestral de 0.05. Se utilizó el análisis estadístico descriptivo, utilizando la herramienta informática SPSS.

\section{RESULTADOS.}

Se presentan los resultados divididos en dimensiones clasificados de acuerdo al cuestionario aplicado. En cada sección se presentan los resultados del análisis descriptivo y de la inferencia estadística. La tabla 1 dimensión estructura organizacional de la variable clima organizacional con la motivación del profesional de enfermería tiene una correlación directa, favorable 55,2\%, desfavorable $42,9 \%$. La relación de la dimensión remuneraciones del clima organizacional con la motivación tiene una correlación directa, se puede evidenciar que los profesionales que tienen una motivación $75,9 \%$ de estos también piensan que las remuneraciones son desfavorables (Tabla 2).En relación a la tabla 3 y 4 con respecto al clima organizacional en su dimensión responsabilidad se puede evidenciar que los profesionales que tienen una motivación desfavorable el $65.5 \%$ de estos también piensan que la responsabilidad es desfavorable; así mismo de este mismo 
porcentaje piensan que el riesgo y toma de decisiones es desfavorable. En las tablas 5 y 6 en la dimensión apoyo se puede evidenciar que los profesionales tienen una motivación desfavorable con el $69 \%$, lo que se observa una correlación directa con la dimensión toma de decisiones, en la tabla 7 referente al clima organizacional y su relación con la motivación de los profesionales de enfermería se obtuvo que de los profesionales que tienen una motivación desfavorable el $89,7 \%$ de estos califican al clima organizacional como desfavorable, similar pasa con los profesionales que tienen una motivación favorable $42,9 \%$ califican al clima organizacional como favorable y medianamente favorable.

Tabla 1. Relación de la dimensión estructura del clima organizacional con la motivación

HRI 20172018

\begin{tabular}{ccccc}
\hline $\begin{array}{c}\text { Estructura } \\
\text { organizacional }\end{array}$ & Favorable & $\begin{array}{c}\text { MOTIVACION } \\
\text { Medianamente favorable }\end{array}$ & Desfavorable & Total \\
\cline { 2 - 4 } Favorable & 16 & 7 & 3 & 26 \\
\cline { 2 - 5 } & $55,2 \%$ & $31,8 \%$ & $42,9 \%$ & $44,8 \%$ \\
\hline $\begin{array}{c}\text { Medianamente } \\
\text { favorable }\end{array}$ & 5 & 14 & 2 & 21 \\
\hline \multirow{2}{*}{ Desfavorable } & $17,2 \%$ & $63,6 \%$ & 2 & $36,2 \%$ \\
\hline & 8 & 1 & $28,6 \%$ & 11 \\
\hline Total & $27,6 \%$ & $4,5 \%$ & 7 & $58 \%$ \\
\hline & 29 & 22 & $100,0 \%$ & $100,0 \%$ \\
\hline
\end{tabular}

Fuente: Base de datos

Tabla 2. Relación de la dimensión remuneraciones del clima organizacional con la motivación HRI $2017-2018$

\begin{tabular}{|c|c|c|c|c|}
\hline \multirow{2}{*}{ Remuneraciones } & \multicolumn{3}{|c|}{ MOTIVACION } & \multirow{2}{*}{ Total } \\
\hline & Favorable & Medianamente favorable & Desfavorable & \\
\hline \multirow{2}{*}{ Favorable } & 4 & 2 & 0 & 6 \\
\hline & $57,1 \%$ & $9,1 \%$ & $0,0 \%$ & $10,3 \%$ \\
\hline \multirow{2}{*}{$\begin{array}{l}\text { Medianamente } \\
\text { favorable }\end{array}$} & 2 & 2 & 7 & 11 \\
\hline & $28,6 \%$ & $9,1 \%$ & $24,1 \%$ & $19,0 \%$ \\
\hline \multirow{2}{*}{ Desfavorable } & 1 & 18 & 22 & 41 \\
\hline & $14,3 \%$ & $81,8 \%$ & $75,9 \%$ & $70,7 \%$ \\
\hline \multirow{2}{*}{ Total } & 7 & 22 & 29 & 58 \\
\hline & $100,0 \%$ & $100,0 \%$ & $100,0 \%$ & $100,0 \%$ \\
\hline
\end{tabular}

Fuente: Base de datos

Tabla 3. Relación de la dimensión responsabilidad del clima organizacional con la motivación HRI $2017-2018$

\begin{tabular}{ccccc}
\hline Responsabilidad & \multicolumn{3}{c}{ MOTIVACION } & \multirow{2}{*}{ Total } \\
\cline { 2 - 4 } Favorable & Favorable & Medianamente favorable & Desfavorable & \\
\cline { 2 - 5 } & 4 & 2 & 0 & 6 \\
\hline Medianamente & $57,1 \%$ & $9,1 \%$ & $0,0 \%$ & $10,3 \%$ \\
\hline favorable & 1 & 7 & 10 & 18 \\
\hline \multirow{2}{*}{ Desfavorable } & $14,3 \%$ & $31,8 \%$ & 19 & $31,0 \%$ \\
\cline { 2 - 5 } & 2 & 13 & $65,5 \%$ & $58,6 \%$ \\
\hline Total & $28,6 \%$ & $59,1 \%$ & 29 & 58 \\
\cline { 2 - 5 } & 7 & 22 & $100,0 \%$ & $100,0 \%$ \\
\hline
\end{tabular}

Fuente: Base de datos 
Tabla 4. Relación de la dimensión riesgo y toma de decisiones del clima organizacional con la motivación HRI 2017 - 2018

\begin{tabular}{ccccc}
\hline $\begin{array}{c}\text { Riesgo y toma de } \\
\text { decisiones }\end{array}$ & Favorable & Medianamente favorable & Desfavorable & Total \\
\cline { 2 - 5 } Favorable & 4 & 2 & 0 & 6 \\
\hline & $57,1 \%$ & $9,1 \%$ & $0,0 \%$ & $10,3 \%$ \\
\hline Medianamente & 1 & 10 & 10 & 21 \\
\hline favorable & $14,3 \%$ & $45,5 \%$ & $34,5 \%$ & $36,2 \%$ \\
\hline Desfavorable & 2 & 10 & 19 & 31 \\
\hline & $28,6 \%$ & $45,5 \%$ & $65,5 \%$ & $53,4 \%$ \\
\hline Total & 7 & 22 & 29 & 58 \\
\hline & $100,0 \%$ & $100,0 \%$ & $100,0 \%$ & $100,0 \%$ \\
\hline
\end{tabular}

Fuente: Base de datos

Tabla 5. Relación de la dimensión apoyo del clima organizacional con la motivación HRI 2017 - 2018

\begin{tabular}{ccccc}
\hline \multirow{2}{*}{ Apoyo } & \multicolumn{3}{c}{ MOTIVACION } & \multirow{2}{*}{ Total } \\
\cline { 2 - 4 } & Favorable & Medianamente favorable & Desfavorable & \\
\cline { 2 - 4 } Favorable & 4 & 2 & 0 & 6 \\
\hline Medianamente & $57,1 \%$ & $9,1 \%$ & $0,0 \%$ & $10,3 \%$ \\
\hline favorable & 2 & 10 & 9 & 21 \\
\hline Desfavorable & $28,6 \%$ & $45,5 \%$ & 20 & $36,2 \%$ \\
\cline { 2 - 4 } & 1 & 10 & $69,0 \%$ & $53,4 \%$ \\
\hline Total & $14,3 \%$ & $45,5 \%$ & 29 & 58 \\
\hline & 7 & 22 & $100,0 \%$ & $100,0 \%$ \\
\hline
\end{tabular}

Fuente: Base de datos

Tabla 6. Relación de la dimensión conflicto del clima organizacional con la motivación HRI 2017 - 2018

\begin{tabular}{ccccc}
\hline \multirow{2}{*}{ Conflicto } & \multicolumn{3}{c}{ MOTIVACION } & \multirow{2}{*}{ Total } \\
\cline { 2 - 4 } Favorable & Favorable & Medianamente favorable & Desfavorable & \\
\cline { 2 - 4 } & 3 & 1 & 0 & 4 \\
\hline Medianamente & $42,9 \%$ & $4,5 \%$ & $0,0 \%$ & $6,9 \%$ \\
\hline favorable & 3 & 8 & 12 & 23 \\
\hline Desfavorable & $42,9 \%$ & $36,4 \%$ & 17 & $39,7 \%$ \\
\cline { 2 - 5 } & 1 & 13 & $58,6 \%$ & $53,4 \%$ \\
\hline Total & $14,3 \%$ & $59,1 \%$ & 29 & 58 \\
\hline & 7 & 22 & $100,0 \%$ & $100,0 \%$ \\
\hline
\end{tabular}

Fuente: Base de datos

Tabla 7. Clima organizacional y su relación con la motivación en profesionales de enfermería del HRI $2017-2018$

\begin{tabular}{ccccc}
\hline CLIMA & \multicolumn{3}{c}{ MOTIVACIÓN } & Total \\
\cline { 2 - 5 } ORGANIZACIONAL & Favorable & Medianamente favorable & Desfavorable & \\
\cline { 2 - 5 } Favorable & 3 & 1 & 0 & 4 \\
\hline Medianamente & $42,9 \%$ & $4,5 \%$ & $0,0 \%$ & $6,9 \%$ \\
favorable & 3 & 4 & 3 & 10 \\
\hline Desfavorable & $42,9 \%$ & $18,2 \%$ & $10,3 \%$ & $17,2 \%$ \\
\hline \multirow{2}{*}{ Total } & 1 & 17 & $89,7 \%$ & $75,9 \%$ \\
\hline & $14,3 \%$ & $77,3 \%$ & 29 & 58 \\
\hline
\end{tabular}




\section{DISCUSIÓN.}

En la investigación se ha encontrado datos relevantes a través de la encuesta realizada a 58 profesionales de enfermería del Hospital Regional de Ica en el año 2017 - 2018 acerca del Clima organizacional y su relación con la motivación laboral.

Referente al clima organizacional en su dimensión estructura organizacional se puede evidenciar que de los profesionales que tienen una motivación desfavorable (7 profesionales de enfermería) el $28,6 \%$ de estos también piensan que la estructura organizacional es desfavorable. Similar pasa en los profesionales que tienen una motivación favorable (29 profesionales de enfermería) el $55,2 \%$ piensan que esta dimensión es también favorable (Tabla 1). En el clima organizacional en su dimensión remuneraciones se puede evidenciar que de los profesionales que tienen una motivación desfavorable (29 profesionales de enfermería) el $75,9 \%$ de estos también piensan que las remuneraciones son desfavorables. Similar pasa en los profesionales que tienen una motivación favorable ( 7 profesionales de enfermería) el $57,1 \%$ piensan que esta dimensión es también favorable (Tabla 2).

Estos resultados son contradictorios al estudio, aunque evaluadas con otras escalas como el de Solís Z. (8), realizada en el 2017 en Lima, donde evidenció percepción favorable según la dimensión cultura organizacional, por la existencia de motivación e identificación hacia los objetivos institucionales, mediante la atención con calidad, solución de problemas con respeto, apoyo mutuo e inteligencia emocional. Sin embargo, según la dimensión diseño organizacional es percibida como desfavorable a excepción de la sub dimensión comunicación que es medianamente favorable, evidenciándose insatisfacción al estilo de mando autoritario, con atmosfera de temor y falta de valores, el favoritismo medie en la toma de decisiones, se sienten discriminados, donde la comunicación cerrada es revertida por la asertiva. Así mismo, según la dimensión potencial humano es percibida como desfavorable debido a las recompensas injustas, carencia de estímulo para el espíritu innovador, tipo de liderazgo de rienda suelta en vez de participativo, la inoperancia de gestión para hacer cumplir las normas técnicas de salubridad laboral.

En la Tabla 3 y 4 con respecto al clima organizacional en su dimensión responsabilidad se puede evidenciar que de los profesionales que tienen una motivación desfavorable (29 profesionales de enfermería) el $65,5 \%$ de estos también piensan que la responsabilidad es desfavorable. Similar pasa en los profesionales que tienen una motivación favorable (7 profesionales de enfermería) el $57,1 \%$ piensan que esta dimensión es también favorable. Referente al clima organizacional en su dimensión riesgo y toma de decisiones se puede evidenciar que de los profesionales que tienen una motivación desfavorable (29 profesionales de enfermería) el $65,5 \%$ de estos también piensan que el riesgo y toma de decisiones es desfavorable. Estos resultados difieren de los profesionales que tienen una motivación medianamente favorable ya que del total de estos (22 profesionales de enfermería) el $45,5 \%$ califican a la dimensión riesgo y toma de decisiones como desfavorable y solo un $9,1 \%$ califica esta dimensión como favorable.

Estos datos son similares al de Chávez D, Ríos D. (3) en su tesis Clima organizacional y satisfacción laboral de las enfermeras en el servicio de cirugía Hospital Víctor Lazarte Echegaray-Trujillo, 2015 donde los resultados muestran un $41 \%$ en el nivel alto y un $59 \%$ en el nivel medio en clima organizacional y en satisfacción laboral se obtuvo un nivel alto con $52 \%$ y un nivel medio con $41 \%$ y nivel bajo $7 \%$.

En la tabla 5 y 6 referente al clima organizacional en su dimensión apoyo se puede evidenciar que de los profesionales que tienen una motivación desfavorable (29 
profesionales de enfermería) el $69 \%$ de estos también piensan que el apoyo es desfavorable. Similar pasa en los profesionales que tienen una motivación favorable (7 profesionales de enfermería) el $57,1 \%$ piensan que esta dimensión es también favorable. En su dimensión conflicto se puede evidenciar que de los profesionales que tienen una motivación desfavorable (29 profesionales de enfermería) el $58,6 \%$ de estos también piensan que el conflicto es desfavorable. Similar pasa en los pacientes que tienen una motivación favorable (7profesionales de enfermería) el $42,9 \%$ piensan que esta dimensión es también favorable.

Estos datos pueden ser contrastados con los realizados por Del Río J, Munares A, Montalvo $H$. (4) en sus tesis Clima organizacional en trabajadores de un hospital general de Ica El clima organizacional percibido por los trabajadores del Hospital tuvo un puntaje promedio de 164 es decir un clima por mejorar. El $12.9 \%$ de trabajadores percibió un clima saludable. De las 11 dimensiones estudiadas, el clima que se percibió fue por mejorar, excepto en identidad, dimensión en la cual se tuvo, en promedio, un clima saludable. Sin embargo, la identidad según grupos ocupacionales, tuvo un clima por mejorar en el grupo de enfermeras, técnicos, otros profesionales y artesanos.

En la tabla 7 respecto al clima organizacional y su relación con la motivación de los profesionales obtuve que de los profesionales que tienen una motivación desfavorable (29 profesionales de enfermería) el $89,7 \%$ de estos también califican al clima organizacional como desfavorable. Similar pasa en los profesionales que tienen una motivación favorable (7 profesionales de enfermería) el $42,9 \%$ califican al clima organizacional como favorable.

Estos resultados se pueden contrastar con Melgar D.(5), investigación y análisis del Clima organizacional en los licenciados de enfermería del Hospital San José de Chincha Marzo 2016 donde encontró un 94\%(47 de licenciados son de sexo femenino, predominan las edades de 46 años a más en el 60\%(30 de ellos), el $70 \%$ (35 de licenciados) son casados, el 36\% (18 licenciados) tienen de 21 años a más de servicio y el $82 \%$ (41 licenciados) son nombrados. Con respecto a la variable Clima organizacional según dimensión comunicación en el $70 \%$ (35 de los licenciados) es medianamente favorable, la dimensión confort en el 66\% (33 licenciados) es medianamente favorable; dimensión estructura es medianamente favorable en el 64\% (32 licenciados), la dimensión identidad en un $62 \%$ (31 de los licenciados) es medianamente favorable, siendo los resultados globales en un 64\% (32 licenciados) medianamente favorable.

\section{CONCLUSIONES.}

1. En esta investigación se acepta la hipótesis de que el clima organizacional está relacionado con la motivación de los profesionales de enfermería, en el Hospital Regional de Ica en el año 2017 - 2018.

2. El clima organizacional en el Hospital Regional de Ica se encuentra en un nivel bajo es decir que no es favorable, por lo que los administradores tienen un buen margen para mejorar esta variable. En relación a las dimensiones de esta variable todas guardan relación con la motivación del profesional significativamente.

3. La motivación de los profesionales de enfermería es desfavorable en un $50,0 \%$, por lo que los tomadores de decisiones pueden mejorar dicho nivel mejorando el clima organizacional de dicho nosocomio.

\section{CORRESPONDENCIA.}

Dra. Zulema Gutiérrez Lazo de la Vega

Correo Electrónico:

zulemagutierrez0902@gmail.com 


\section{REFERENCIAS BIBLIOGRAFICAS.}

1. Salazar G. Clima organizacional y grado de motivación. $2^{-a}$ ed. México: Ed. PrenticeHall; 2009.

2. Vera M. Clima organizacional de enfermería en los hospitales regionales del Instituto de Previsión Social. Tesis Pregrado. Asunción, Paraguay, Facultad de Enfermería, Universidad Nacional de Asunción; 2014.

3. Chávez D, Ríos D. Clima organizacional y satisfacción laboral de las enfermeras en el servicio de cirugía Hospital Víctor Lazarte Echegaray- Trujillo, 2015.Tesis Pregrado. Trujillo, Perú. Facultad de Enfermería Universidad Privada Antenor Orrego; 2015.

4. Del Río J, Munares A, Montalvo H. Clima organizacional en trabajadores de un hospital general de Ica. Rev. Med Panacea. 2013; 3(1).

5. Melgar D. Clima organizacional en los licenciados de enfermería del Hospital San José de Chincha Marzo 2016. Tesis Pregrado. Ica, Perú. Facultad de Enfermería. Universidad Privada San Juan Bautista;.2016.
6. Chiavenato, I. Administración de recursos humanos. Bogotá, Colombia: Ed. Mac Graw- Hill. Interamericana, S.A; 2012.

7. Cruz F. Gestión integrada de recursos humanos. España: Ediciones Deusto. S.A.; 2000.

8. Solís Z. Clima organizacional en los trabajadores del Hospital de Baja Complejidad Vitarte, 2013. Tesis Master. Lima, Perú. Facultad de Medicina, Universidad Nacional de San Marcos; 2017.

Recibido: 02/10/2019

Aprobado para publicación: 14/03/2020 